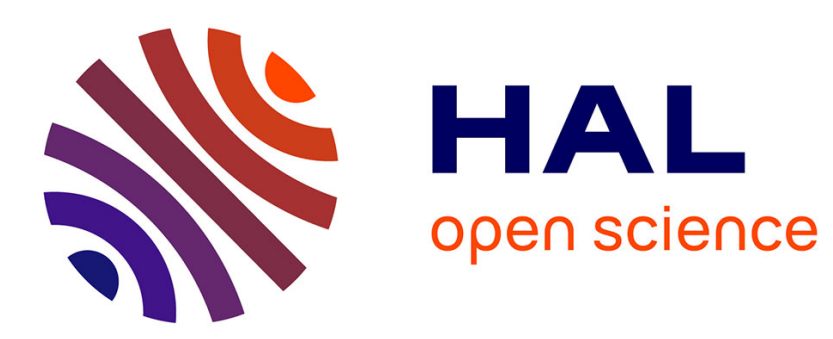

\title{
Hybrid Statistical-Structural On-line Chinese Character Recognition with Fuzzy Inference System
}

\author{
Adrien Delaye, Sébastien Macé, Eric Anquetil
}

\section{To cite this version:}

Adrien Delaye, Sébastien Macé, Eric Anquetil. Hybrid Statistical-Structural On-line Chinese Character Recognition with Fuzzy Inference System. International Conference on Pattern Recognition, IAPR, Dec 2008, Tampa, Florida, United States. inria-00322952

\section{HAL Id: inria-00322952 \\ https://hal.inria.fr/inria-00322952}

Submitted on 19 Sep 2008

HAL is a multi-disciplinary open access archive for the deposit and dissemination of scientific research documents, whether they are published or not. The documents may come from teaching and research institutions in France or abroad, or from public or private research centers.
L'archive ouverte pluridisciplinaire HAL, est destinée au dépôt et à la diffusion de documents scientifiques de niveau recherche, publiés ou non, émanant des établissements d'enseignement et de recherche français ou étrangers, des laboratoires publics ou privés. 


\title{
Hybrid Statistical-Structural On-line Chinese Character Recognition with Fuzzy Inference System
}

\author{
Adrien Delaye - Sébastien Macé - Eric Anquetil \\ IRISA - INSA, Campus Universitaire de Beaulieu, 35042 Rennes Cedex, France \\ \{adrien.delaye,sebastien.mace,eric.anquetil\}@irisa.fr
}

\begin{abstract}
In this paper, we propose an original hybrid statistical-structural method for on-line Chinese character recognition. We model characters thanks to fuzzy inference rules combining morphological and contextual information formalized in a homogeneous way. For that purpose, we define a set of primitives modeling all the stroke classes that can be found in handwritten Chinese characters. Thus, each analyzed stroke can be classified as primitive without any segmentation process. Inference rules are built from the coupling of a priori information about the primitives constituting the characters and automatic modeling of their relative positioning. The fuzzy inference system aggregates these rules for decision making. First experiments validate this method with a recognition rate of $97.5 \%$ on a subset of Chinese characters.
\end{abstract}

\section{Introduction}

Online Chinese character recognition has been widely studied for the last thirty years. Beyond the complexity of characters, the huge number of classes (more than 6000 daily used) and the great variability in the order and the shapes of strokes induced by different writing styles make their recognition an intricate problem. Unlike Latin characters, Chinese characters have structural characteristics that must be taken into account for their recognition. It is generally admitted that future methods should combine both structural and statistical information in order to overcome recognition rates offered by methods that follow either a statistical or a structural approach [5]. In our lab, we have a strong background on Latin character recognition [1], and we are working on transposing some of our experience to the problem of Chinese character recognition. In this paper, we introduce a hybrid method associated with a simple modeling relying on fuzzy sets theory and we demonstrate its validity for Chinese character recognition. As a first step toward a more general Chinese character recognition system, we focus on the problem of recognizing Chinese radicals. Radicals constitute a subset of Chinese characters. Their shapes are rather simple, and they are the elementary components of more complicated characters. Indeed, any Chinese character, no matter how complex, can be described as a composition of various radicals. Our recognition system aims at recognizing radicals that are written in a lowconstrained, stroke-order free style. The difficult task of segmenting an input sequence into elementary units, common to most of the structural approaches, is here avoided thanks to the definition of our modeling primitives. That is, we model primitives so as to directly describe the cursive strokes usually drawn when writing characters in a natural fluent style. Then radicals are described by fuzzy inference rules that model morphological information (shape of the primitives constituting the radical) and information about spatial context (relative positioning between these primitives). Decision is processed by merging these two kinds of information in a fuzzy inference system. This approach, though very simple and intuitive, leads to promising results. In the following, we introduce the principles of our method and the global architecture of the system. Learning process is described in section 3 and the method we use for evaluation of relative positioning is presented in section 4. Then we present how this model is exploited and expose some experimental results in section 6 before concluding.

\section{Modeling and general architecture}

In this section, we present the principles of the recognition system we propose, by introducing first the primitives of our structural description and then the fuzzy inference system that models the Chinese radicals. 


\subsection{Stroke primitives}

Chinese characters are theoretically described as a canonical combination of fundamental strokes. Nevertheless, in freely handwritten characters, we observe strong shape variations : strokes are often connected, lines become curved, etc. Therefore, finding the canonical decomposition of fluently written characters into such fundamental strokes is a complex segmentation problem. To overcome this, we propose to categorize all the types of individual raw (possibly cursive) strokes as the primitives of our modeling. This choice is justified by the strong stability of such primitives among different writers.

In [4], Kim et al. also propose to exploit such primitives. They use an unsupervised technique to define a primitive dictionary. On the contrary, we have chosen to define this dictionary manually, because we need these primitives to be meaningful for using them later to describe the radicals. We built a dictionary of primitives from a significant Chinese character database : it defines 31 primitives, as presented in figure 1(a). Primitives numbered from 1 to 20 are fundamental Chinese strokes, whereas others result from merging or warping of fundamental strokes.
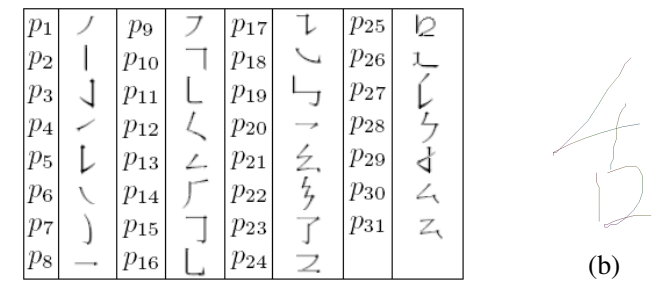

(b)

(a)

Figure 1. (a) primitives dictionary (b) radical "SHE"

A character $R_{i}$ can be decomposed into such primitives $p_{i_{j}}$ :

$$
R_{i} \rightarrow p_{i_{1}} p_{i_{2}} \ldots p_{i_{n}}
$$

This way, the "SHE" radical presented figure 1(b) can be described as $R_{S H E} \rightarrow p_{2} p_{2} p_{13} p_{24}$.

\subsection{Radical modeling by fuzzy inference system}

The modeling of each radical is formalized by a fuzzy inference rule of the form :

$$
\underbrace{\left(F_{i_{1}} \wedge \ldots \wedge F_{i_{n}}\right)}_{\text {shapes }} \wedge \underbrace{\left(C_{i_{1}} \wedge \ldots \wedge C_{i_{m}}\right)}_{\text {context }} \Rightarrow R_{i}
$$

where $F_{i_{j}}$ are fuzzy conditions relative to the shapes of expected strokes and $C_{i_{j}}$ are fuzzy conditions relative to the expected between-strokes positioning within the radical $R_{i}$. Given an input sequence to be classified, activation degrees for these conditions are calculated with respect to fuzzy prototypes that are automatically learnt (see section 3). During the recognition process, rules in which the number of primitives is not coherent with the number of strokes in the input sequence are filtered out. Finally, the remaining rules are completely homogenous because they all consist of the same number of fuzzy conditions. They are aggregated in a Takagi-Sugeno fuzzy inference system [6] of order 0 and the decision is taken by a max-product fuzzy inference method (see subsection 5.2).

\section{Learning}

Fuzzy inference rules modeling the radicals such as (2) result from a priori information on how the radical $R_{i}$ can be decomposed into primitives and automatic modeling of relative position between these primitives. Its complete formalisation requires modeling $n$ shape fuzzy prototypes and $n-1$ positioning fuzzy prototypes, because our relative positioning is evaluated for each primitive relatively to a unique reference primitive (see details in section 4). We present here how fuzzy prototypes of each type are learnt and how they are used for evaluating fuzzy conditions activation degree in (2).

\subsection{Learning of shape fuzzy prototypes}

The primitives defined in 2.1 are modeled by fuzzy prototypes. Features are extracted from training samples, leading to 20-dimensional feature vectors. The features are the same as the ones used in RESIF, an efficient online recognition system for Latin alphabet based languages [1]; they include features such as bending, relative dimensions, number of downward paths, loops, etc. Feature vectors are then clustered into fuzzy prototypes by unsupervised fuzzy clustering (Fuzzy CMeans) independently processed for each class, so that resulting prototypes only model intrinsic characteristics of the class.

For an input stroke $X_{k}$ of unknown primitive type, the activation degree of a fuzzy condition $F_{j}$ is computed by :

$$
F_{j}\left(X_{k}\right)=\max _{P_{r} \in P_{j}}\left(\frac{1}{1+d_{Q_{r}}\left(X_{k}, \mu_{r}\right)}\right)
$$

where $P_{j}$ denotes the set of fuzzy prototypes involved in the modeling of primitive $j$, and $\mu_{r}$ and $Q_{r}$ are the mean vector and covariance matrix of the prototype $P_{r}$. 


\subsection{Learning of positioning fuzzy prototypes}

For each rule under the form (2), consisting of $n$ primitives, $n-1$ fuzzy prototypes are learnt to model the relative positioning of strokes within the radical. Assuming that the vector $v^{X_{1}}\left(X_{j}\right)$ describes the position of input stroke $X_{j}$ relatively to reference input stroke $X_{1}$, the activation degree of a fuzzy condition $C_{i_{j}}\left(X_{j}\right)$ is evaluated by the same function as used in (3) :

$$
C_{i_{j}}\left(X_{j}\right)=\frac{1}{1+d_{Q_{r}}\left(v^{X_{1}}\left(X_{j}\right), \mu_{r}\right)}
$$

$\mu_{r}$ and $Q_{r}$ being the parameters of the fuzzy prototype $P_{r}$ modeling the expected relative positions.

\section{Fuzzy evaluation of relative positioning}

The relative positioning of the primitives composing a radical is essential for its recognition. Most of existing approaches modelize such relations as either adjacency, or intersection, or parallelism, etc, often in a crisp manner. However, relative positioning of handdrawn strokes can be ambiguous. Thus, we exploit the fuzzy set framework to evaluate such relations.

For that purpose, we use the method proposed by Bloch in [2] and adapted to the on-line context in [3]. It makes it possible to evaluate the relative directional relationships of an analyzed object relatively to a reference object. This method can be divided into two steps [2]. First, we define a fuzzy landscape around the reference object : it is a fuzzy set that defines the membership value of each point of the space regarding the relation under examination. Then, we compare the analyzed object to the fuzzy landscape in order to evaluate how well the object matches with the relation.

\subsection{Defining a fuzzy landscape}

Defining a fuzzy landscape consists in defining the fuzzy set $\mu_{\alpha}(R)$, that represents the adequacy of any point $P$ of the space $S$ with the directional relation defined by the angle $\alpha$ and relatively to the reference object $R$.

Let us denote by $P$ any point of the space $S$, and by $Q$ any point in $R$. Let $\beta(P, Q)$ be the angle between the vector $\overrightarrow{Q P}$ and the direction $\vec{u}_{\alpha}$, computed in $[0, \pi]$. $\beta(P, Q)$ is given by the equation :

$$
\beta(P, Q)=\arccos \left(\frac{\overrightarrow{Q P} \cdot \vec{u}_{\alpha}}{\|\overrightarrow{Q P}\|}\right), \text { and } \beta(P, P)=0
$$

For each point $P$, we determine the point $Q$ of the reference object $R$ leading to the smallest angle $\beta$, denoted by $\beta_{\min }(P, R)$.
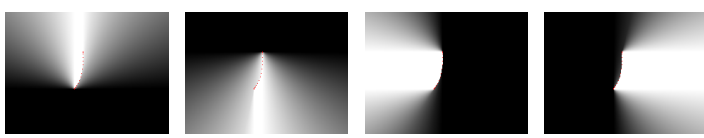

Figure 2. Fuzzy landscapes (white color depicting highest membership values)

The fuzzy landscape $\mu_{\alpha}(R)$ can then be defined as :

$$
\mu_{\alpha}(R)(P)=\max \left(0,1-\frac{2 \beta_{\min }(P, R)}{\pi}\right)
$$

Figure 2 presents examples of fuzzy landscapes defined around a reference stroke; they correspond to the relative directional relationships above, below, left_of and right_of defined relatively to a reference stroke.

\subsection{Comparing an object with a landscape}

Once the fuzzy landscape is defined, we want to evaluate the degree to which an analyzed object $A$ satisfies the corresponding relation. For such purpose, we use the average value proposed in [2] :

$$
M_{\alpha}^{R}(A)=\frac{1}{|A|} \sum_{P \in A} \mu_{\alpha}(R)(P)
$$

where $|A|$ is the cardinality of $A$. Figure 3 presents examples of $M_{\alpha}^{R}(A)$ values.

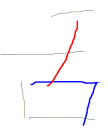

\begin{tabular}{l|r} 
Direction & Degree \\
\hline above & 0.05 \\
below & 0.83 \\
left_of & 0.21 \\
right_of & 0.86
\end{tabular}

Figure 3. Positioning vector for a primitive (in blue) relatively to a reference (in red)

\subsection{Exploitation in our method}

We use the presented method to evaluate the relative positioning of a Chinese primitive relatively to another one. We define a positioning vector containing four degrees corresponding to the adequacy to four different directions (above, below, left_of, right_of, see figure 3).

Two measures of relative dimensions of analyzed primitive relatively to reference primitive are also included in the vector. This 6-dimensional positioning vector $v^{R}(A)$ is robust with respect to scale variations and character stretching.

\section{Exploitation process}

In this section, we focus on the recognition process. In two distinct steps, we first select consistent rules and 
then process the inference on this reduced set of fuzzy rules.

\subsection{Rule selection}

We consider the analysis of a stroke sequence $T=t_{1} \ldots t_{n}$. As introduced previously, no segmentation process is necessary. Thus, only fuzzy inference rules involving exactly $n$ primitives are selected.

Each stroke is compared to each shape fuzzy prototype : this leads to the computation of $F_{c}\left(t_{i}\right)$ for each stroke $t_{i}$ relatively to each class of primitives $c$. A rejection threshold is set, below which it is not pertinent to consider the primitive hypothesis. Then, if none of the analyzed strokes matches a primitive class $c$, all productions involving $c$ are filtered out. This significantly reduces the number of rules to be evaluated.

\subsection{Rule evaluation and decision making}

As all remaining rules involve the same number of primitives, we can compute an activation degree for each and compare them homogeneously through the fuzzy inference system. For each rule (2), $n$ shape scores (evaluated by (3)) and $n-1$ position scores (evaluated by (4)) are merged with a t-norm fuzzy operator, resulting in a global score :

$$
\begin{aligned}
& S\left(R_{a}\right)=F_{a_{1}}\left(u_{1}\right) \otimes \quad \ldots \otimes F_{a_{n}}\left(u_{n}\right) \\
& \otimes C_{a_{2}}\left(u_{2}\right) \otimes \ldots \otimes C_{a_{n}}\left(u_{n}\right)
\end{aligned}
$$

where $\left\{u_{1}, \ldots, u_{n}\right\}$ is the permutation of $\left\{t_{1}, . ., t_{n}\right\}$ that maximizes $S\left(R_{a}\right)$ : this insures the stroke-order free property of our system.

Global scores of all rules are then compared, and the conclusion of the best satisfied fuzzy rule is considered as the eventually recognized radical.

\section{Experimentations and results}

Experiments have been conduced on a subset of the database constituted by the CASIA ${ }^{1}$.

Learning of the fuzzy prototypes modeling the shapes of the primitives was led on a training database of 1000 samples per class, written by 40 writers. We wrote 48 rules describing 35 radicals, each constituted of two to six primitive strokes. Relative positions of the primitives in a radical were learnt with 75 samples for each rule, written by the same 40 writers. Test of the global system was conduced on 1200 radical examples ( 25 per rule) written by 20 other writers. The recognition rate is $97,5 \%$, and the average number of activated rules in the fuzzy inference system is about 12.3.

\section{Conclusion}

In this paper, we presented a method for the recognition of Chinese radicals. We first defined a dictionary of indivisible primitives corresponding to the strokes that can be found in Chinese characters : this allows to avoid a difficult segmentation step. Then, it is only necessary to write rules about the primitives that constitute each considered symbol. Information about primitive shapes and their relative positioning are added thanks to an automatic learning process. These morphological and contextual information are modeled under an homogeneous formalism and merged in the decisionmaking process by a fuzzy inference system.

The presented method is well adapted to the recognition of radicals written in a current style. It is also omniwriter and stroke-order-free. First experiments validate the approach : recognition rates reach $97.5 \%$. Future works will aim at reducing the complexity of recognition process using a more accurate reject strategy and defining criterion for automatic identification of reference primitive stroke in order to allow extending this method to the frame of complete Chinese characters set.

\section{Acknowledgments}

The authors would like to thank Professor G. Lorette for his precious advice as well as Professor C.-L. Liu for letting us use his database.

\section{References}

[1] E. Anquetil and H. Bouchereau. Integration of an on-line handwriting recognition system in a smart phone device. In Proceedings of ICPR'02, pages 192-195, 2002.

[2] I. Bloch. Fuzzy relative position between objects in image processing: a morphological approach. IEEE Transactions on PAMI, 21(7):657-664, 1999.

[3] F. Bouteruche, S. Macé, and E. Anquetil. Fuzzy relative positioning for on-line handwritten stroke analysis. In Proceedings of IWFHR'06, pages 391-396, 2006.

[4] H. Kim, J. Jung, and S. Kim. On-line Chinese character recognition using ART-based stroke classification. Pattern Recognition Letters, 17(12):1311-1322, 1996.

[5] C.-L. Liu, S. Jaeger, and M. Nakagawa. Online recognition of Chinese characters: the State-of-the-Art. IEEE Transactions on PAMI, 26(2):198-213, 2004.

[6] T. Takagi and M. Sugeno. Fuzzy identification of systems and its applications to modeling and control. IEEE Transactions on Systems, Man, and Cybernetics, 15:116-132, 1985.

\footnotetext{
${ }^{1}$ Chinese Academy of Science, Institute of Automation
} 